Artículo

\title{
Caracterización del pasto mombaza como materia prima para producir bioetanol
}

\begin{abstract}
Joel Ventura Ríos ${ }^{1}$
Mario Alberto Santiago Ortega ${ }^{2}$

Iliana Barrera-Martínez ${ }^{3 \S}$

Perpetuo Álvarez Vázquez ${ }^{1}$

Pedro Carrillo López ${ }^{1}$

José Amador Honorato Salazar ${ }^{4}$

${ }^{1}$ Universidad Autónoma Agraria Antonio Narro. Calzada Antonio Narro núm. 1923, Buena vista, Saltillo, Coahuila, México. CP. 25315. (joelventur@gmail.com; carrillo2lpz@gmail.com; perpetuo.alvarezv@uaaan.edu.mx). ${ }^{2}$ Colegio de Postgraduados-Campus Montecillo. Carretera Federal México-Texcoco km 36.5, Montecillo, Texcoco, Estado de México. CP. 56230. Tel. 5959520279. (saomar87@gmail.com). ${ }^{3}$ CONACYT-CIATEJ. Camino Arenero 1227, El Bajío, Zapopan, Jalisco. CP. 45019. (ibarrera@ciatej.mx). ${ }^{4}$ Campo Experimental-INIFAP. Carretera Federal México-Puebla km 56.5, San Martinito, Tlahuapan, Puebla, México. CP. 74100. (ahonorato650@gmail.com).
\end{abstract}

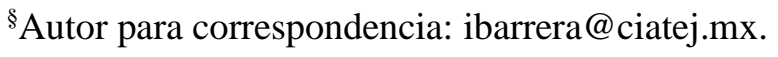

\section{Resumen}

El objetivo de este estudio realizado en 2017 fue caracterizar el pasto mombaza (Megathyrsus maximus) para evaluar su potencial como materia prima para la producción de biocombustible líquido (bioetanol). La producción anual de biomasa, el poder calorífico, la composición química y el rendimiento teórico de bioetanol fueron determinadas en cuatro frecuencias de corte (30, 60, 90 y 120 d después del rebrote). Los datos se analizaron con el procedimiento GLM (SAS) y las medias de los tratamientos se compararon con la prueba de Tukey $(p \leq 0.05)$. La mayor producción de biomasa, poder calorífico, producción de energía, producción de bioetanol, FDN, LDA y hemicelulosa se obtuvieron en la frecuencia de corte de $120 \mathrm{~d}$ con $11 \mathrm{Mg} \mathrm{ha}^{-1} \mathrm{año}^{-1} ; 16.1 \mathrm{MJ} \mathrm{kg}^{-1}$; 178.4 GJ ha ${ }^{-1}$ año $^{-1} ; 238.2 \mathrm{~L} \mathrm{Mg}^{-1} \mathrm{MS}$ y 68.6, 6.5, 23.3\%, respectivamente. No obstante, el mayor contenido de celulosa y FDA se encontraron en la frecuencia de corte de $90 \mathrm{~d}$, con 41.2 y $47.4 \%$, respectivamente. Los valores más altos de humedad, PC y cenizas se encontraron en la frecuencia de corte de $30 \mathrm{~d}$ con valores de $8.2,10.4$ y $12.1 \%$, respectivamente, mientras que el contenido de EE fue mayor en la frecuencia de corte de $60 \mathrm{~d}(1.6 \%)$. De acuerdo con los resultados obtenidos en este estudio el pasto mombaza (Megathyrsus maximus) puede ser considerado como una materia prima atractiva para la producción de bioetanol en climas tropicales.

Palabras clave: biocombustible, biomasa, composición química pasto, valor calorífico.

Recibido: enero de 2021

Aceptado: marzo de 2021 


\section{Introducción}

Los sistemas de producción de pastos en el trópico subhúmedo de México dependen de las condiciones climáticas a largo del año, afectando el rendimiento de biomasa y modificando su composición química. En la región costera del Golfo de México el crecimiento de las plantas está determinado por tres épocas en el año: 1) época de lluvias; de junio a octubre, cuando la temperatura y agua, favorecen el crecimiento de las plantas; 2) época de nortes; de noviembre a febrero, en la que disminuye el crecimiento de la mayoría de las especies forrajeras, por las bajas temperaturas y alta nubosidad; y 3) época seca; de marzo a mayo, cuando el rendimiento de biomasa se reduce drásticamente por la casi nula precipitación (Hernández et al., 1990).

Ante esto, para aumentar la producción de carne y leche en las zonas tropicales de México se han introducido especies forrajeras con alto potencial para utilizarse en sistemas de corte o pastoreo. De ellas, destacan las variedades del género Megathyrsus maximus, [Sin. Panicum maximum, (Simon y Jacobs, 2003)] que son especies de mayor potencial de crecimiento y producción de materia seca (MS) por hectárea (Ramírez et al., 2009) además, de gran adaptabilidad creciendo desde el nivel del mar hasta más de $1000 \mathrm{~m}$ de altitud.

La constitución de los pastos está determinada por los componentes químicos de la pared y su contenido celular (Mertens, 1997). En las gramíneas la celulosa y hemicelulosa representan aproximadamente $70 \%$ del total de biomasa (MS) y su contenido de lignina varía entre 10-30\%. Los azúcares se unen a la lignina a través de enlaces covalentes hidrofóbicos, lo que le confiere alta resistencia a cualquier tratamiento para ser separados (Weijde et al., 2013). Por otro lado, la composición química de las plantas también varía de acuerdo con la especie, tipo de tejido, etapa de crecimiento y condiciones de crecimiento (Wongwatanapaiboon et al., 2012).

El bioetanol producido a partir de biomasa lignocelulósica puede usarse como oxigenante de la gasolina, mejorando la calidad del aire ya que reduce la emisión de $\mathrm{CO}_{2}$. La Agencia Internacional de Energía (2018), en el año 2016 reportó una producción de 13760.81 MMtep de bioetanol. Los mayores productores fueron China (17.2\%), Estados Unidos de América (13.9\%), Rusia (10\%), Arabia Saudita (4.9\%) e India (4.1\%), mientras que México solo aporto 1.3\% [https://www.iea.org/reports/world-energy-balances-2019].

La composición química de los pastos tropicales y su concentración de azúcares estructurales, pueden ser aprovechadas para obtención de bioetanol de segunda generación y otras formas de energía. Sin embargo, en México los estudios sobre la producción de etanol a partir de biomasa y su relación con composición química, rendimientos de biomasa, edad de corte, estado fenológico y especie de plantas son muy limitados (Rueda et al., 2016; Santiago et al., 2016; Ventura et al., 2017). El objetivo del presente estudio fue evaluar el rendimiento de biomasa, composición química, caracterización energética y rendimiento teórico de bioetanol del pasto mombaza (Megathyrsus maximus) cosechado a cuatro frecuencias de corte (FC).

\section{Materiales y métodos}

El rendimiento de biomasa se evaluó en el sitio experimental 'Papaloapan' del Instituto Nacional de Investigaciones Forestales, Agrícolas y Pecuarias (INIFAP), a $18^{\circ} 06^{\prime}$ latitud norte y $95^{\circ} 31^{\prime}$ longitud oeste y $65 \mathrm{msnm}$, en Cd. Isla, Veracruz, México, con clima $\mathrm{A}_{\text {wo }} \mathrm{y}$ temperatura media de 
$25.7{ }^{\circ} \mathrm{C}$ (García, 2004); el suelo es acrisol órtico, franco-arenoso, con pH de 4 a 4.7, pobre en materia orgánica, nitrógeno, calcio, potasio y contenidos medios a altos de fósforo y magnesio (Enríquez y Romero, 1999). Las variables evaluadas fueron rendimiento de biomasa, materia seca, proteína cruda (PC), fibra detergente neutro (FDN), fibra detergente acido (FDA), celulosa, hemicelulosas, lignina digestible en acido (LDA), extracto etéreo (EE), cenizas, poder calorífico, humedad y rendimiento de bioetanol.

\section{Establecimiento de parcelas}

La siembra se efectuó el 22 de julio de 2017, en surcos con separación de $0.5 \mathrm{~m}$, en parcelas experimentales de $5 \mathrm{~m}$ de ancho por $16 \mathrm{~m}$ de longitud, con tres repeticiones. Se aplicaron dos fertilizaciones a 43 y 112 d después de la siembra, con la fórmula: 120-80-00 kg ha-1 de $\mathrm{N}_{\text {y }} \mathrm{P}_{2} \mathrm{O}_{5}$.

\section{Rendimiento de biomasa (Mg MS ha' ${ }^{-1}$ ano $^{-1}$ )}

La acumulación de biomasa se determinó por unidad de superficie para cada FC: 30, 60, 90 y 120 d después del corte de homogeneización (ddch) en muestreos destructivos por un año. En cada parcela se ubicó al azar en cinco ocasiones, un cuadrante de metal de $\left(1 \mathrm{~m}^{2}\right)$ y se cortó el forraje total, a $20 \mathrm{~cm}$ de altura residual. La biomasa cosechada, se pesó en balanza de precisión (Ohaus, Mod. GT-4000; $6.200 \mathrm{~kg} \pm 0.1 \mathrm{~g}$ ). La submuestra se pesó y se secó en una estufa de convección forzada (Felisa, Mod. FE-243A), a $55^{\circ} \mathrm{C}$ durante 72 h y se calculó la MS.

\section{Preparación de las muestras para análisis}

Las determinaciones químicas y el poder calorífico se realizaron con muestras seca molidas (molino Wiley ${ }^{\circledR}$ PA, USA) y tamizadas con mallas no. $40(0.42-1 \mathrm{~mm})$ y no. $60(0.25-0.42$ $\mathrm{mm})$.

\section{Análisis químico}

Las muestras se incineraron durante $2 \mathrm{~h}$ a $600{ }^{\circ} \mathrm{C}$ para obtener el contenido de materia orgánica y cenizas (Norma ASTM D 1102-84; 2012). La concentración PC se midió por el método Kjeldahl (N x 6.25) y el EE en extractor Soxhlet (AOAC, 1990). Las concentraciones de FDN, FDA (Van Soest et al., 1991) y de LDA (Goering y Van Soest, 1970), se obtuvieron secuencialmente en al analizador de fibras ANKOM ${ }^{200 ®}$ (Ankom Technology, Fairport, NY, USA), mediante el uso de bolsas filtro Ankom ${ }^{\circledR}$ F57 con un tamaño de poro de 30 micrones. Para determinar la FDN se utilizó sulfito de sodio $\left(\mathrm{Na}_{2} \mathrm{SO}_{3}\right)$ y $\alpha$-amilasa para remover el nitrógeno y el almidón de la muestra, respectivamente. Las hemicelulosas y celulosa se calcularon mediante la diferencia entre FDN y FDA, y entre FDA y lignina, respectivamente.

\section{Poder calorífico}

Se determinó en un calorímetro de bomba adiabática (Isoperibol, Parr 1266), de acuerdo con la norma ASTM (E711), a $30 \pm 0.5^{\circ} \mathrm{C}$, con pastillas comprimidas de $1 \mathrm{~g}$ máximo. El contenido de humedad se determinó en una balanza térmica Ohaus MB45 ${ }^{\circledR}$. Se realizaron cinco determinaciones por muestra y 15 repeticiones por FC. 


\section{Rendimiento teórico de bioetanol}

El rendimiento teórico de bioetanol (RTE) se determinó con base a las reacciones de hidrólisis y de transformación de azúcares a etanol, tomando en cuenta su estequiometria. Se utilizaron las fórmulas propuestas por Badger (2002); Dien (2010).

Celulosa hemicelulosa: $\mathrm{RTE}_{\mathrm{C}}=\mathrm{C} \times \mathrm{C}_{\mathrm{g} / \mathrm{c}} \times \mathrm{E}_{\mathrm{CC}} \times \mathrm{R}_{\mathrm{et}} \times \mathrm{E}_{\mathrm{fg}} \times \mathrm{D}_{\mathrm{et}} \mathrm{RTE}_{\mathrm{H}}=\mathrm{H} \times \mathrm{H}_{\mathrm{x} / \mathrm{h}} \times \mathrm{E}_{\mathrm{CH}} \times$ $\mathrm{R}_{\mathrm{et}} \times \mathrm{E}_{\mathrm{fx}} \times \mathrm{D}_{\mathrm{et}}$. Total. RTE $=\mathrm{RTE}_{\mathrm{c}}+\mathrm{RTE}_{\mathrm{H}}$.

Donde: $\quad$ RTE: $\mathrm{LMg}^{-1} \mathrm{MS} ; \quad \mathrm{C}: \mathrm{kg}_{\text {celulosa }} \mathrm{Mg}^{-1} \mathrm{Mg}_{\text {biomasa }}^{-1} ; \quad \mathrm{H}: \mathrm{kg}_{\text {hemicelulosa }} \mathrm{Mg}_{\text {biomasa }}$; $\mathrm{C}_{\mathrm{g} / \mathrm{c}}$ : concentración de glusosa $\left(1.111 \frac{\mathrm{kg}_{\text {glucosa }}}{\mathrm{kg}_{\text {celulus }}}\right) ; \quad \mathrm{C}_{\mathrm{h} / \mathrm{x}}$ : concentración de xilosa $\left(1.136 \frac{\mathrm{kg}_{x \text { xilosa }}}{\mathrm{kg}_{\text {hemicelulosa }}}\right)$; $\mathrm{E}_{\mathrm{cc}}$ : eficiencia de conversión de celulosa (0.76);

$\mathrm{E}_{\mathrm{ch}}$ : eficiencia de conversión de hemicelulosa (0.9);

RTE: rendimiento estiquiométrico de etanol $\left(0.511 \mathrm{~kg}_{\text {etanol }} / \mathrm{kg}_{\text {glucosa }} ; 0.511 \mathrm{~kg}_{\text {etanol }} / \mathrm{kg}_{\text {xilosa }}\right)$; $\mathrm{E}_{\mathrm{fg}}$ : eficiencia fermentativa de glucosa $(0.75) ; \mathrm{E}_{\mathrm{fx}}$ :eficiencia fermentativa de xilosa (0.5); $\mathrm{D}_{\mathrm{et}}$ : densidad de etanol $\left(0.78 \mathrm{Mg} \mathrm{m}^{-3}\right)$.

El rendimiento teórico anual de bioetanol que produce 1 ha de mombaza se calculó para cada FC, multiplicando el rendimiento teórico de bioetanol por unidad de biomasa por el rendimiento anual de biomasa.

\section{Análisis estadístico}

Los datos se analizaron como un diseño completamente al azar, donde se consideró la frecuencia de corte del pasto mombaza como tratamientos $(30,60,90$ y 120) con tres repeticiones por cada tratamiento. Se realizó un análisis de varianza (Anova) para identificar el efecto de la frecuencia de corte sobre las variables de respuesta mediante el uso del procedimiento GLM/SAS y las medias de tratamientos se compararon con la prueba de Tukey $(p \leq 0.05)$ utilizando SAS para Windows versión 9.3 (SAS, 2011).

\section{Resultados y discusión}

\section{Rendimiento de biomasa}

El rendimiento de biomasa se incrementó linealmente a medida que la planta avanzo en su madurez fisiológica, la mayor producción de biomasa se encontró en la FC de $120 \mathrm{~d}\left(11 \mathrm{Mg} \mathrm{ha}^{-1}\right.$ año), quien fue diferente (pc 0.05) al resto de las frecuencias de corte (Cuadro 1). La FC de 120 d superó en $23.5,11.1$ y $6 \%$, a las frecuencias de 30,60 y 90 d, respectivamente. Verdecía et al. (2009), reportaron rendimientos de 3.6 y $6.4 \mathrm{Mg} \mathrm{MS} \mathrm{ha}^{-1}$ a 30 y 60 días, respectivamente, valores menores a lo encontrado en este estudio en las mismas frecuencias de cosecha.

Por otro lado, Ramírez et al. (2009) evaluó la producción de biomasa del pasto mombaza y reportó variaciones de 9.7 a $20.6 \mathrm{Mg}$ MS ha-1 de 3 a 7 semanas en época de lluvias. El rendimiento de biomasa en el experimento fue diferente en cada FC, esto se puede atribuir a las condiciones de crecimiento, factores asociados con la fisiología de la planta y en particular a la temperatura y la humedad los factores que tienen mayor influencia en la producción de biomasa a través del año. 
Cuadro 1. Rendimiento de biomasa y producción de energía del pasto del pasto mombaza (Megathyrsus maximus) cosechado a diferentes frecuencias de corte.

\begin{tabular}{ccccc}
\hline $\begin{array}{c}\text { Frecuencia de corte } \\
(\text { días })\end{array}$ & $\begin{array}{c}\text { Rendimiento } \\
\left(\mathrm{Mg} \mathrm{MS} \mathrm{ha}^{-1} \mathrm{año}^{-1}\right)\end{array}$ & $\begin{array}{c}\text { Poder calorífico } \\
\left(\mathrm{MJ} \mathrm{kg}^{-1}\right)\end{array}$ & $\begin{array}{c}\text { Producción de } \\
\text { energía } \\
\left(\mathrm{GJ} \mathrm{ha}^{-1} \mathrm{año}^{-1}\right)\end{array}$ & $\begin{array}{c}\text { Humedad } \\
(\%)\end{array}$ \\
\hline 30 & $8.9 \mathrm{~d}$ & $15.8 \mathrm{~b}$ & $142.1 \mathrm{c}$ & $8.2 \mathrm{a}$ \\
60 & $9.9 \mathrm{c}$ & $13.7 \mathrm{~d}$ & $136.2 \mathrm{~d}$ & $8 \mathrm{~b}$ \\
90 & $10.4 \mathrm{~b}$ & $14.5 \mathrm{c}$ & $151.6 \mathrm{~b}$ & $7.7 \mathrm{c}$ \\
120 & $11 \mathrm{a}$ & $16.1 \mathrm{a}$ & $178.4 \mathrm{a}$ & $8 \mathrm{ab}$ \\
Media & 10 & 15 & 152.1 & 8 \\
EE & 0.83 & 0.2 & 3.3 & 0.04 \\
\hline
\end{tabular}

$\mathrm{EE}=$ error estándar. Letras diferentes muestran diferencias entre cortes (Tukey, $p \leq 0.05$ ).

\section{Poder calorífico, producción de energía y contenido de humedad}

El mayor contenido de poder calorífico se encontró en la FC de 120 d (16.1 $\left.\mathrm{MJ} \mathrm{kg}^{-1}\right)$, valor estadísticamente diferente ( $p \leq 0.05$ ) a las demás FC (Cuadro 1). En este estudio el rango de valores de poder calorífico fue de 13.7 a $16.1 \mathrm{MJ} \mathrm{kg}^{-1}$, siendo la FC de 30 y $120 \mathrm{~d}$ las que mayor concentración de energía mostraron.

Los valores obtenidos en el experimento son menores a los de Santiago et al. (2016); Mohammed et al. (2015), quienes reportaron en promedio $16.5 \mathrm{MJ} \mathrm{kg}^{-1}$ en pasto Toledo (Urochloa brizantha) y pasto Napier (Pennisetum purpureum). Por otro lado, se ha reportado para Switchgrass y bagazo de sorgo dulce 17.3 y $13.7 \mathrm{MJ} \mathrm{kg}^{-1}$, respectivamente (Shankar, 2015; Nhuchhen y Salam, 2012).

Por otra parte, la producción de energía por hectárea estuvo en función del rendimiento de materia seca. La mayor producción energía por hectárea (178.4 GJ ha-1 año $\left.{ }^{-1} ; p \leq 0.05\right)$ se encontró en la FC de 120 d (Cuadro 1). Este valor resultó 31, 25.5 y 17.6\% mayor a los encontrados en las FC de 60, 30 y 90 d, respectivamente. La mayor producción de energía fue menor a lo publicado por Santiago et al. (2016) quienes reportan $466.6 \mathrm{GJ} \mathrm{ha}^{-1} \mathrm{año}^{-1}$ en la misma FC en pasto Toledo (Urochloa brizantha).

El contenido de humedad más alto se presentó en la FC de $30 \mathrm{~d}(8.2 \%)$, el cual fue similar ( $p \geq$ $0.05)$ a la FC de $120 \mathrm{~d}$, pero diferente $(p \leq 0.05)$ a las demás (Cuadro 1$)$. Se ha reportado que contenidos elevados de humedad por unidad de masa, reducen la eficiencia de la combustión, debido a que gran parte del calor liberado se usa para evaporar el agua en lugar de la reducción química del material (Nhuchhen y Salam, 2012).

\section{Proteína cruda}

El contenido proteína cruda disminuyó a medida que se incrementó la FC. La mayor concentración de proteína cruda se encontró a 30 d $(10.4 \%)$, valor superior $(p \leq 0.05)$ al resto de las FC (Cuadro 2). El contenido de proteína cruda disminuyo en 37.5, 41.1 y $51 \%$, al pasar de la menor FC (30 d) a los 60, 90 y 120 d, respectivamente. Al respecto, se ha demostrado que la concentración de PC en las plantas está relacionada con la fertilidad del suelo. Sin embargo, en etapas tempranas la demanda de este nutriente es elevado en la planta y va disminuyendo a medida que aumenta la biomasa aérea (Ramírez et al., 2013). 
Cuadro 2. Composición química del pasto mombaza (Megathyrsus maximus) cosechado a diferentes frecuencias de corte.

\begin{tabular}{ccccccc}
\hline \multirow{2}{*}{$\begin{array}{c}\text { Frecuencia de } \\
\text { corte(días) }\end{array}$} & PC & FDN & FDA & LDA & Extracto etéreo & Cenizas \\
\cline { 2 - 7 } & $10.4 \mathrm{a}$ & $60.4 \mathrm{c}$ & $43 \mathrm{~d}$ & $4.2 \mathrm{~d}$ & $1.2 \mathrm{c}$ & $12.1 \mathrm{a}$ \\
60 & $6.5 \mathrm{~b}$ & $60.2 \mathrm{~d}$ & $44.7 \mathrm{c}$ & $4.7 \mathrm{c}$ & $1.6 \mathrm{a}$ & $11 \mathrm{~b}$ \\
90 & $5.5 \mathrm{c}$ & $62.5 \mathrm{~b}$ & $47.4 \mathrm{a}$ & $6.2 \mathrm{~b}$ & $1.1 \mathrm{~d}$ & $10.6 \mathrm{c}$ \\
120 & $5.1 \mathrm{~d}$ & $68.6 \mathrm{a}$ & $45.2 \mathrm{~b}$ & $6.5 \mathrm{a}$ & $1.5 \mathrm{~b}$ & $10.6 \mathrm{c}$ \\
Media & 6.9 & 62.9 & 45.1 & 5.4 & 1.3 & 11.1 \\
EE & 2.2 & 3.6 & 1.6 & 1 & 0.2 & 0.6 \\
\hline
\end{tabular}

$\mathrm{PC}=$ proteína cruda $; \mathrm{FDN}=$ fibra detergente neutro; $\mathrm{FDA}=$ fibra detergente acida; $\mathrm{LDA}=$ lignina digestible en acido. $\mathrm{EE}=$ error estándar. Letras diferentes muestran diferencias entre cortes (Tukey, $p \leq 0.05$ ).

Por su parte, Rojas et al. (2018) reportaron la diminución en la concentración de PC del 34 y 52\%, al pasar de 35 a 49 d y de 35 a 63 d de corte, respectivamente en pasto Cobra (Brachiaria hibrido BR02/1794). Los resultados obtenidos en este estudio presentan una tendencia similar a la observada por Rojas et al. (2018). Ventura et al. (2019) reportaron disminuciones de $34 \%$ al pasar de 30 a 60 d y $42 \%$ al pasar de 30 a 90 días de corte en pasto Maralfalfa (Cenchrus purpureus Schumach.) Morrone. Por otro lado, se reportan concentraciones de proteína cruda de 8.7, 9 y 8.5\% para el cultivar Mombaza, Privilegio y Tanzania, respectivamente (Ortega et al., 2011).

\section{Fibra detergente neutra y fibra detergente ácida}

Los mayores contenidos de FDN y FDA ( $p \leq 0.05)$ se encontraron en la FC de $120 \mathrm{~d}(68.6 \%)$ y 90 d (47.4\%), respectivamente (Cuadro 2). El uso de las fibras de los pastos en la industria del bioetanol no está documentado; sin embargo, tienen uso importante en la industria de alimentos para animales, la pulpa y obtención de papel (Rueda et al., 2016). Ortega et al., (2011) reportaron en Megathyrsus maximus cv. Mombaza 68.9 y $47.1 \%$ y en pasto Tanzania 73.6 y $46.3 \%$ para FDN y FDA, respectivamente.

Por otro lado, Alves et al. (2014) reportaron 67.9\% de FDN y 39.3\% de FDA en Brachiaria brizanta cv. Marandu y $66.1 \%$ de FDN y $37.1 \%$ de FDA en Brachiaria brizanta cv. Xaraes. Al respecto, Mertens (1997) menciona que la composición química de los pastos y otras fuentes forrajeras está influenciada por los compuestos que forman la pared y contenido celular en la planta en el momento de cosecha. Estos compuestos se modifican por la adición de fertilizantes, precipitación y radiación solar.

\section{Lignina digestible en ácido}

Al contrario que la PC y cenizas, el mayor contenido de LDA se encontro en la FC de $120 \mathrm{~d}(6.5 \%)$ el cual fue diferente $(p \leq 0.05)$ al resto de las FC (Cuadro 2). La concentración de LDA a $120 \mathrm{~d}$ superó en 55, 38 y 5\% a la de 30, 60 y 90 d, respectivamente. Los resultados obtenidos son similares 
a los obtenidos por Wongwatanapaiboon et al. (2012), quienes reportaron 4.4\% en pasto pangola (Digitaria decumbens), 5.6\% en atratum (Paspalum atratum) y 4\% en pasto guineo (Megathyrsus maximus).

Coêlho et al. (2018) reportaron 1, 1.3 y 1.9\% en Megathyrsus maximus, Digitaria pentzii y Urochloa mosambicensis, respectivamente. Por otro lado, en maderas coníferas y latifoliadas la concentración de LDA es mayor que en pastos o herbáceas. Sharma et al. (2016) reportaron concentraciones de 30 a $60 \%$ en abeto (Abies alba Mill) y pino (Pinus spp.), mientras que Limayen y Ricke (2012) reportan 20 a $25 \%$ en maderas duras o latifoliadas.

\section{Extracto etéreo}

La mayor concentración de extracto etéreo se encontró a los 60 d (1.6\%), valor estadísticamente diferente $(p \leq 0.05)$ al resto de las FC (Cuadro 2). El intervalo de contenido de extracto etéreo fue de 1.1 a $1.6 \%$, similar a los resultados obtenidos en otras investigaciones. Por ejemplo, Kondo et al. (2015) reportaron 2.8, 2, 2.1 y $1.8 \%$ en Andropogun gayanus, Urochloa decumbens, Megathyrsus maximus y Urochloa mutica, respectivamente. En estudios de bioenergía es importante cuantificar grasas liposolubles, pigmentos y otros tipos de sustancias que pueden alterar los análisis posteriores (Sluiter et al., 2008).

\section{Cenizas}

El mayor contenido de cenizas se registró a los 30 d (12.1\%), el cual fue estadísticamente diferente $(p \leq 0.05)$ a las demás (Cuadro 2). Por lo que, conforme aumentó la edad de rebrote la concentración de cenizas disminuyo. Normalmente el contenido de cenizas y materia inorgánica es alto en etapas tempranas de desarrollo de la planta, pero este compuesto no ofrece ningún aporte energético (Mohameed et al., 2015).

El contenido de cenizas es mayor en herbáceas y residuos agrícolas, respecto a otros materiales lignocelulósicos (Limayen y Ricke, 2012). Santiago et al. (2016) reportaron 7\% en pasto Toledo (Urochloa brizantha) mientras que Rueda et al. (2016) reportaron en cultivares del género (Cenchrus purpureus Schumach.) Morrone, 8.8, 9.4, 10.6 y 10.7\% en Caña africana, King grass, Taiwán y CT115, respectivamente.

\section{Celulosa}

La mayor concentración de celulosa se encontró a los $90 \mathrm{~d}\left(41.2 \% ; 4.2 \mathrm{Mg} \mathrm{ha}^{-1} \mathrm{año}^{-1}\right)$, el cual fue diferente ( $p \leq 0.05$ ) a las demás FC (Cuadro 3). A los $90 \mathrm{~d}$ el contenido de celulosa fue 24, 8.3 y $0.2 \%$ mayor al encontrado a los 30,60 y $120 \mathrm{~d}$, respectivamente. Los resultados obtenidos en este estudio son similares a los reportados con otros materiales lignocelulosicos; por ejemplo, Jahirul et al. (2012) reportaron $24 \%$ de celulosa en Miscanthus sp., mientras que, Wongwatanapaiboon et al. (2012) y Santiago et al. (2016) registraron 34.5 y $42.1 \%$ en pasto Ruzi (Brachiaria ruziziensis) y Toledo (Urochloa brizantha), respectivamente. Como se ha reportado, la composición química y en particular los azucares estructurales en los pastos, se modifican a medida que la planta avanza en su madurez fisiológica (Lima et al., 2014). 
Cuadro 3. Concentración de celulosa y hemicelulosas del pasto mombaza (Megathyrsus maximus) cosechado a diferentes frecuencias de corte.

\begin{tabular}{cccccc}
\hline \multirow{2}{*}{ Frecuencia de corte (días) } & \multicolumn{2}{c}{ Celulosa } & & \multicolumn{2}{c}{ Hemicelulosas } \\
\cline { 2 - 3 } \cline { 5 - 6 } & $(\%)$ & $\left(\mathrm{Mg} \mathrm{ha}^{-1} \mathrm{año}^{-1}\right)$ & & $(\%)$ & $\left(\mathrm{Mg} \mathrm{ha}^{-1} \mathrm{año}^{-1}\right)$ \\
\hline 30 & $38.7 \mathrm{c}$ & $3.4 \mathrm{~d}$ & & $17.4 \mathrm{~b}$ & $1.5 \mathrm{~b}$ \\
60 & $40 \mathrm{~b}$ & $3.9 \mathrm{c}$ & & $15.5 \mathrm{c}$ & $1.5 \mathrm{~b}$ \\
90 & $41.2 \mathrm{a}$ & $4.2 \mathrm{a}$ & & $15.1 \mathrm{~d}$ & $1.5 \mathrm{~b}$ \\
120 & $38.7 \mathrm{c}$ & $4.2 \mathrm{a}$ & & $23.3 \mathrm{a}$ & $2.5 \mathrm{a}$ \\
Media & 39.6 & 4 & & 17.8 & 1.8 \\
EE & 1.1 & 0.07 & & 3.5 & 0.09 \\
\hline
\end{tabular}

$\mathrm{EE}=$ error estándar. Letras diferentes muestran diferencias entre cortes (Tukey, $p \leq 0.05$ ).

\section{Hemicelulosas}

La FC 120 d presentó el mayor contenido de hemicelulosas (23.3\%; $2.5 \mathrm{Mg} \mathrm{ha}^{-1} \mathrm{año}^{-1}$ ) el cual fue estadísticamente diferente $(p \leq 0.05)$ al resto de las FC (Cuadro 3). La concentración de este componente a 120 d fue 54, 50 y $34 \%$ mayor a la encontrada a los 90,60 y 30 d, respectivamente. Se ha reportado concentraciones de hemicelulosas de 22.1, 27 y 28\% en pasto Elefante (Cenchrus purpureus Schumach.) Morrone, bagazo de caña (Saccharum officinarum) y sorgo (Sorghum biocolor L.; Moench), respectivamente (Carroll y Somerville, 2009; Weijde et al., 2013; Mohameed et al., 2015). Las paredes de gramíneas y plantas herbáceas contienen menor concentración de hemicelulosas que residuos agrícolas (25-50\%), maderas de coníferas (22-29\%) y latifoliadas (25-40\%), según Limayen y Ricke (2012).

\section{Rendimiento teórico a bioetanol}

El mayor rendimiento teórico de etanol celulósico se encontró a 120 d (238.2 $\mathrm{L} \mathrm{Mg}^{-1} \mathrm{MS} ; 2640 \mathrm{~L}$ $\left.\mathrm{ha}^{-1} \mathrm{año}^{-1}\right)$. Este valor representó una diferencia estadística representativa con respecto al resto de la FC ( $p \leq 0.05$ ) (Cuadro 4). El rendimiento a $120 \mathrm{~d}$ fue mayor en 35, 22 y $14.5 \%$ al encontrado a los 30, 60 y $90 \mathrm{~d}$, respectivamente.

Cuadro 4. Rendimiento teórico de bioetanol del pasto mombaza (Megathyrsus maximus) cosechado a diferentes frecuencias de corte.

\begin{tabular}{ccc}
\hline \multirow{2}{*}{ Frecuencia de corte(días) } & \multicolumn{2}{c}{ Producción de bioetanol } \\
\cline { 2 - 3 } & $\left(\mathrm{L} \mathrm{Mg}^{-1} \mathrm{MS}\right)$ & $\left(\mathrm{L} \mathrm{ha}^{-1} \mathrm{año}^{-1}\right)$ \\
\hline 30 & $218.5 \mathrm{c}$ & $1954.8 \mathrm{~d}$ \\
90 & $217.5 \mathrm{~d}$ & $2156.8 \mathrm{c}$ \\
120 & $221.1 \mathrm{~b}$ & $2304.9 \mathrm{~b}$ \\
Media & $238.2 \mathrm{a}$ & $2640.5 \mathrm{a}$ \\
EE & 223.8 & 2264.2 \\
\hline
\end{tabular}

$\mathrm{EE}=$ error estándar. Letras diferentes muestran diferencias entre cortes (Tukey, $p \leq 0.05$ ). 
Algunos de los estudios conducidos en México con materiales lignocelulósicos para la producción de bioetanol reportan, $272 \mathrm{~L} \mathrm{Mg}^{-1} \mathrm{MS}$ con Urochloa brizanta cv. Toledo (Santiago et al., 2016) y con pasto maralfalfa (Cenchrus purpureus Schumach.) Morrone $3851 \mathrm{~L} \mathrm{ha}^{-1}$ (Ventura et al., 2017). Por otro lado, Lima et al. (2014) reportaron rendimientos de 282.6, 283.3 y $285.7 \mathrm{~L} \mathrm{Mg}^{-1} \mathrm{MS}^{\text {en }}$ bagazo de caña, Eucaliptus grandis bark y Megathyrsus maximus, respectivamente.

Las estimaciones del rendimiento anual son similares a lo publicado por Wongwatanapaiboon et al. (2012) quienes reportaron 2561 y $2621 \mathrm{~L} \mathrm{ha}^{-1}$ en pasto Napier y King grass (Cenchrus purpureus Schumach.) Morrone. En otras investigaciones con materias primas de primera generación, Zhao et al. (2009) y Somerville et al. (2010) reportaron rendimientos de 2967 a 13 $032 \mathrm{~L} \mathrm{ha}^{-1}$ en sorgo (Sorghum bicolor (L.) Moench y $3800 \mathrm{~L} \mathrm{ha}^{-1}$ en maíz (Zea mays L.), respectivamente.

De acuerdo con los resultados obtenidos en este estudio el pasto mombaza representa una materia prima prometedora para la producción de bioetanol. Sin embargo, se deben de tomar en cuenta otros aspectos para asegurarse de la pertinencia técnica, económica y ambiental del uso de este cultivo energético para la producción de bioetanol.

\section{Conclusiones}

El incremento en el tiempo de cosecha permitió un mayor rendimiento de biomasa aérea del pasto mombaza (Megathyrsus maximus), guardando una relación directa con el contenido de lignina y producción de bioetanol. De acuerdo con los resultados encontrados no se pudo establecer una relación directa entre el tiempo de crecimiento y el poder calorífico, producción de energía, FDN, hemicelulosa, porcentaje de humedad, PC y cenizas.

Por otra parte, no se detectó una relación estrecha entre la frecuencia de corte y la FDA, extracto etéreo y concentración de celulosa. No obstante, los resultados del rendimiento de biomasa y las características químicas indican que el pasto mombaza (Megathyrsus maximus), es una opción técnicamente viable para la producción de bioetanol en zona tropical.

\section{Literatura citada}

Agencia Internacional de Energía. 2020. IEA world energy balance. 2019. https://www.iea.org/ regions/north-america.

ASTM. 2012. American Society for Testing and Materials. Annual book of ASTM standards, biological effects and environmental fate; biotechnology. ASTM E870. West Cornshohocken, PA, USA. 11(06):96-97.

Alves, M. G.; Aparecida-Phino, C. K.; Da-Costa S. E.; Soares, E. P.; Flavio, N. J.; Goncalves, R. M.; Bezerra, F. P.; Guimarães, S. J. F. and Gomes, G. W. 2014. Yield and chemical composition of Brachiaria forage grasses in the offseason after corn harvest. Am. J. Plant Sci. 5(5):933-941. https://doi.org/10.4236/ajps.2014.57106.

AOAC. 1990. Association of Official Analytical Chemists. Protein (crude) determination in animal feed. Official Methods of Analysis. 15 ${ }^{\text {th }}(\mathrm{Ed})$. Helrich, K. Arlington. VA, USA. $72-74 \mathrm{pp}$. 
Badger, P. C. 2002. Ethanol from cellulose: a general review. In: trends in new crops and new uses. Janick, J. and Whipkey, A. (Ed). 630/J33t. ASHS Press. Alexandria, Virginia. USA. 17-21 pp. http://large.stanford.edu/publications/coal/references/docs/badger.pdf.

Carroll, A. and Somerville, C. 2009. Cellulosic biofuels. Annu. Rev. Plant Biol. 60(1):165-182. https://doi.org/10.1146/annurev.arplant.043008.092125.

Coêlho, J. J.; Leão-M. A. C.; Ferreira, D. M. V.; Batista, D. J. J. C.; Vieira-Da, C. M. and Lira, M. A. 2018. Prediction of the nutritional value of grass species in the semiarid region by repeatability analysis. Pesq. Agropec. Bras. 53 (3):378-385. https://doi.org/10.1590/s0100$204 \times 2018000300013$.

Enríquez, Q. J. F. y Romero, M. J. 1999. Tasa de crecimiento estacional a diferentes edades de rebrote de 16 ecotipos de Brachiaria spp. en Isla, Veracruz. Agrociencia. 33(2):141-148. https://www.colpos.mx/agrocien/96-99/332.pdf.

Dien, B. S. 2010. Mass balances and analytical methods for biomass pre-treatment experiments. In: biomass to biofuels: strategies for global industries. Vertès, A. N.; Qureshi, A.; Blaschek, H. and Yukawa, H. (Ed). Wiley. Chichester, United Kingdom. 213-231 pp.

García, E. 2004. Modificación al sistema de clasificación climática de Köppen. $4^{\text {a }}$ (Ed). Instituto de Geografía. Universidad Nacional Autónoma de México (UNAM). México. 217 p.

Goering, H. K. and Van-Soest, P. J. 1970. Forage fiber analyses: apparatus, reagents, procedures, and some applications (No. 379). Agricultural Research Service, US Department of Agriculture.

Hernández, T.; Valles, B. y Castillo, E. 1990. Evaluación de gramíneas y leguminosas forrajeras en Veracruz, México. Pasturas Trop. 12(3):29-33.

Jahirul, M. I.; Rasul, M. G.; Chowdhury, A. A. and Ashwath, N. 2012. Biofuels production through biomass pyrolysis a technological review. Energies. 5(12):4952-5001. https://doi.org/10.3390/en5124952.

Kondo, M.; Yoshida, M.; Loresco, M.; Lapitan, M. R.; Herrera, V. J. R.; Del Barrio, N.; Uyeno, Y.; Matsui, H. and Fujihara, T. 2015. Nutrient contents and in vitro ruminal fermentation of tropical grasses harvested in wet season in the Philippines. Adv. Anim. Vet. Sci. 3(12):694-699. http://dx.doi.org/10.14737/journal.aavs/2015/3.12.694.699.

Lima, M. A.; Gomez, L. D.; Steele-King, C. G.; Simister, R.; Bernardinelli, O. D.; Carvalho, M. A.; Rezende, C. A.; Labate, C. A.; de Azevedo, E. R.; McQueen-Mason S. J. and Polikarpov, I. 2014. Evaluating the composition and processing potential of novel sources of Brazilian biomass for sustainable biorenewables production. Biotechnol. Biofuels. 7(1):1-19. https://doi.org/10.1186/1754-6834-7-10.

Limayem, A. and Ricke, S. C. 2012. Lignocellulosic biomass for bioethanol production: current perspectives, potential issues and future prospects. Prog. Energy Combust. Sci. 38(4):449467. https://doi:10.1016/j.pecs.2012.03.002.

Mertens, R. D. 1997. Creating a system for meeting the fiber requirements of dairy cows. J. Dairy Sci. 80(7):1463-1481. https://doi:10.3168/jds.S0022-0302(97)76075-2.

Mohammed, Y. I.; Abakr, A. Y.; Kazi, K. F.; Yusup, S.; Alshareef, I. and Chin, A. S. 2015. Comprehensive characterization of napier grass as a feedstock for thermochemical conversion. Energies. 8(5):3403-3417. https://doi.org/10.3390/en8053403.

Ortega, G. R.; Castillo, G. E.; Rodríguez, J. J.; Escobar, H. R.; Ocaña, Z. E. and de la Mora, B. V. 2011. Nutritive quality of ten grasses during the rainy season in a hot -humid climate and ultisol soil. Trop. Subtrop. Agroecosys. 13(3):481-491. 
Nhuchhen, D. R. and Salam, P. A. 2012. Estimation of higher heating value of biomass from proximate analysis: a new approach. Fuel. 99(8):55-63. https://doi.org/10.1016/ j.fuel.2012.04.015.

Ramírez, R. O.; Hernández, G. A.; Carneiro-Da, S. S.; Pérez, P. J.; Enríquez, Q. J. F.; Quero, C. A. R.; Herrera, H. J. G. and Cervantes, N. A. 2009. Acumulación de forraje, crecimiento y características estructurales del pasto mombaza (Panicum maximum Jacq.) cosechado a diferentes intervalos de corte. Téc. Pec. Méx. 47(2):203-213.

Ramírez, O. S.; Domínguez, D. D.; Salmerón, Z. J. J.; Villalobos, V. G. y Ortega, G. J. A. 2013. Producción y calidad del forraje de variedades de avena en función del sistema de siembra y de la etapa de madurez al corte. Rev. Fitotec. Mex. 36(4):395-403.

Rojas, G. A. R.; Torres, S. N.; Maldonado, P. M.; Sánchez, S. P.; García, B. A.; Mendoza, P. S.; Álvarez, V. P.; Herrera, P. J. y Hernández, G. A. 2018. Curva de crecimiento y calidad del pasto Cobra (Brachiaria hibrido br02/1794) a dos intensidades de corte. Agroproductividad. 11(5):34-38. https://www.researchgate.net/profile/Adelaido_Rojas_ Garcia/publication/326426605.

Rueda, J. A.; Ortega, J. E.; Hernández, G. A.; Enríquez, Q. J. F.; Guerrero, R. J. D. and Carrillo, A. R. Q. 2016. Growth, yield, fiber content and lodging resistance in eight varieties of Cenchrus purpureus (Schumach.) Morrone intended as energy crop. Bio. Bioenergy. 88(5):59-65. https://doi.org/10.1016/j.biombioe.2016.03.007.

SAS. 2011. Statistical Analysis System. Institute. User's Guide of SAS Release 9.3. SAS Institute Inc., Cary, NC. USA. 550 p.

Santiago, O. M. A.; Honorato, S. J. A.; Quero, C. A. R.; Hernández, G. A.; López, C. C. and López, G. I. 2016. Biomasa de Urochloa brizantha cv. Toledo como materia prima para la producción de bioetanol. Agrociencia. 50(6):711-726.

Sharma, V.; Sharma, S. and Kuila, A. 2016. A review on current technological advancement of lignocellulosic bioethanol production. J. Appl. Biotechnol. Bioeng. 1(2):1-7. https://doi.org/10.15406/jabb.2016.01.00011.

Shankar, T. J. 2015. Comparison of chemical composition and energy property of torrefied switchgrass and corn stover. Front. Energy Res. 3(46):1-11. https://doi.org/10.3389/ fenrg.2015.00046.

Somerville, C.; Youngs, H.; Taylor, C.; Davis, S. C. and Long, S. P. 2010. Feedstocks for lignocellulosic biofuels. Science. 329(5993):790-792. https://doi.org/10.1126/ science. 1189268.

Simon, K. B. and Jacobs, S. W. L. 2003. Megathyrsus, a new generic name for Panicum subgenus Megathyrsus. Austrobaileya. 6(3): 571-574.

Sluiter, A.; Ruiz, R.; Scarlata, C.; Sluiter, J. and Templeton, D. 2008. Determination of Extractives in Biomass. Technical Report NREL/TP-510-42619.

Van-Soest, P. J.; Robertson, J. B. and Lewis, B. A. 1991. Methods for dietary fiber, neutral detergent fiber, and non-starch polysacharides in relation to animal nutrition. J. Dairy Sci. 74(10):3583-3589. https://doi.org/10.3168/jds.S0022-0302(91)78551-2.

Weijde, T.; Kamei, A. C. L.; Torres, A. F.; Vermerris, W.; Dolstra, O.; Visser, R. G. F. and Trindade, M. L. 2013. The potential of C4 grasses for cellulosic biofuel production. Plant Sci. 4(107):1-18. https://doi.org/10.3389/fpls.2013.00107.

Verdecía, D. M.; Ramírez, J. L.; Leonard, I. y García, F. 2009. Potencialidades agroproductivas de dos cultivares de Panicum maximum (c.v Mombaza y Uganda) en la provincia Granma. Rev. Electron de Vet. 10(5):1-9. 
Ventura, R. J.; Honorato, S. J. A.; Hernández, G. A.; Aburto, A. J. A.; Vaquera, H. H. y Enríquez, Q. J. F. 2017. Composición química y rendimiento de biomasa de maralfalfa para producción de bioetanol de segunda generación. Rev. Méx. Cienc. Agríc. 8(1):215-221. https://doi.org/10.29312/remexca.v8i1.85.

Ventura, R. J.; Reyes, V. I.; García, S. A.; Muñoz, G. C.; Muro, R. A.; Maldonado, P. M.; Rojas, G. A. y Cruz, H. A. 2019. Rendimiento, perfil nutrimental y de fermentación ruminal in vitro de pasto maralfalfa (Cenchrus purpureus Schumach.) Morrone a diferentes frecuencias de corte en clima cálido. Acta Universitaria. 29(1):1-11. https://doi.org/10.15174/au.2019.2204.

Zhao, Y. L.; Dolat, A.; Steinberger, Y.; Wang X.; Osman, A. and Xie, G. H. 2009. Biomass yield and changes in chemical composition of sweet sorghum cultivars grown for biofuel. Field Crops Res. 111(1-2):55-64. https://doi.org/10.1016/j.fcr.2008.10.006.

Wongwatanapaiboon, J.; Kangvansaichol, K.; Burapatana, V.; Inochanon, R.; Winayanuwattikun, P.; Yongvanich, T. and Chulalaksananukul, W. 2012. The potential of cellulosic ethanol production from grasses in Thailand. J. Biomed Biotechnol. 303(748):1-10. https://doi.org/10.1155/2012/303748. 